\title{
Cambio de técnica de punción de la fístula arteriovenosa nativa a partir de los hallazgos ecográficos
}

\author{
Raúl Darbas-Barbé, Ramón Roca-Tey, Caty Cabot Franco, Gloria Tinto Iborra, Celia Balada Sancho, María \\ Jesús Taboada Álvarez, Cristina Domínguez Orgaz, Lourdes Moliner Calderón, Sara Tornel García, Tania \\ Curado Soto, Alba Luque Tinto y Laura Román García
}

Área nefrológica del Hospital de Mollet. Mollet del Vallés. Barcelona. España

\section{Resumen}

Introducción: La técnica de punción en área debe evitarse siendo la técnica escalonada de primera elección en la mayoría de los casos.

Objetivo: Identificar a los pacientes portadores de una fístula arteriovenosa nativa que se puncionan mediante la técnica del área y que son susceptibles de cambiarla por la técnica escalonada a partir de los hallazgos obtenidos mediante la exploración mediante ecografía doppler.

Pacientes y métodos: Los enfermos del estudio son enfermos tratados mediante hemodiálisis asistida (HD) tres veces por semana que cumplen los siguientes criterios de inclusión: Edad superior a 18 años, prevalentes en programa de HD crónica, con acceso vascular permanente tipo fístula arteriovenosa nativa humeral o radial y puncionado mediante la técnica del área. EI método de exploración de la fístula arteriovenosa nativa fue la ecografía doppler portátil. Los parámetros ecográficos se determinaron por duplicado y fueron los siguientes: flujo sanguíneo de arteria nutricia $(\mathrm{ml} / \mathrm{min})$, diámetro y profundidad del segmento de vena arterializada que no se punciona $(\mathrm{cm})$. Se diseñó un estudio transversal y observacional que se efectuó durante el mes de enero de 2016.

Resultados: De un total de 63 pacientes prevalentes en HD crónica, se registraron: 24 fistulas arteriovenosas protésicas o catéteres y 39 fístula arteriovenosa nativa. De éstas, sólo 9 se puncionaban mediante la técnica de área (14,4\%): 6 humerobasílicas y 3 humeroperforantes (edad media $73,0 \pm 13,3$ años, $77,7 \%$ con alguna comorbilidad). Respecto al resultado de los parámetros ecográficos, se objetivó una media de $0.7 \pm 0.3 \mathrm{~cm}$ de diámetro y $0.5 \pm 0.3 \mathrm{~cm}$ de profundidad en el segmento de vena arterializada que no se punciona y el flujo medio de la arteria humeral fue de $1309,9 \pm 966 \mathrm{ml} /$ minuto. En la mayoría de los mismos fue posible utilizar directamente la técnica escalonada gracias a la ampliación de la zona puncionable de la vena arterializada utilizando la ecografía doppler. Y en dos enfermos, fue imprescindible la superficialización previa de la misma. Por tanto, todos los casos de técnica del área pueden ser potencialmente cambiados por la punción escalonada.

\section{Conclusión:}

1) Se ha registrado una baja incidencia de utilización de la técnica de punción en área en nuestra Unidad de HD.

2) Según los resultados de nuestro estudio, es factible cambiar la técnica de punción en área por escalonada en todos los casos a partir de los parámetros morfológicos y funcionales obtenidos en la fístula arteriovenosa nativa.

3) La ecografía doppler portátil utilizada "in situ" en la sala de HD es una herramienta valiosa que permite optimizar la técnica de punción de la fístula arteriovenosa nativa.

PALABRAS CLAVE

- ECOGRAFÍA DOPPLER

- PUNCIÓN ESCALONADA

- PUNCIÓN EN ÁREA

- ACCESO VASCULAR

- FÍSTULA ARTERIOVENOSA NATIVA

- HEMODIÁLISIS 


\section{Changing the arteriovenous fistula (avf) needling technique based on the ultrasound findings}

\begin{abstract}
Introduction: Most AVFs for hemodialysis (HD) should be punctured by the rope-ladder method. The area technique for AVF cannulation should be avoided whenever possible. On the other hand, cannulation guided by Doppler ultrasound (DU) can increase the success of difficult cannulation procedures.
\end{abstract}

Objective: Identify the patients dialyzed through an AVF using the area technique for cannulation that are susceptible for changing to the rope-ladder technique based on the DU findings.

\section{Patients and methods:}

- During January-2016 we have performed a crosssectional observational study in the prevalent HD patients dialyzed through an AVF with two needles using the area technique for cannulation.

- All patients were explorer by DU using GE Logic $\mathrm{E}$ (linear array 7.5 MHz) device. The following parameters were explored by DU: blood flow rate of the feeding artery $(\mathrm{ml} / \mathrm{min})$, diameter and depth of the arterialized vein segment that never was punctured $(\mathrm{cm})$. All parameters by DU were measured in duplicated and the results were averaged. All determinations by DU were performed by the same explorer.

Results: From 63 prevalent HD patients, 39 patients had AVF (61.9\%) and, of them, only 9 patients (14.3\%) were dialyzed through an AVF using the area technique for cannulation (mean age $73.0 \pm 13.3$ years, $11.1 \%$ diabetic nephropathy, time on HD 53.6 26.9 months). All AVF were brachial artery-based AVF (6 brachiobasilic and 3 braquioperforating). The ultrasongraphic findings were the following: mean diameter $0.7 \pm 0.3 \mathrm{~cm}$, mean depth $0.5 \pm 0.3 \mathrm{~cm}$ and mean flow $1309.9 \pm 966 \mathrm{ml} / \mathrm{min}$. Only two cases had the arterialized vein in more than $0.6 \mathrm{~cm}$ of depth and, therefore, they will need will perform a vein transposition procedure for apply the rope-ladder technique for cannulation. In the remaining cases $(n=7)$, is possible to perform the rope-ladder technique immediately using DU by increasing the puncture zone extension in the arterialized vein.

\section{Conclusions:}

1. It has been recorded a low incidence of area technique for AVF needling in our HD Unit.

2. It is feasible to change the needling AVF technique from area to rope-ladder technique in all cases by means of the morphological and functional parameters recorded in the AVF.

3. The portable DU used "in situ" in the HD Unit is a valuable tool that allows to change the technique for AVF cannulation.

\section{KEYWORDS}

- DOPPLER ULTRASOUND

- TROPE-LADDER TECHNIQUE

- AREA TECHNIQUE

- VASCULAR ACCESS

- AVF

- HEMODIALYSIS

\section{Introducción}

El acceso vascular (AV) es básico y vital para los pacientes con enfermedad renal terminal (ERCT). EI conseguir un AV adecuado y mantener su función a lo largo del tiempo es imperativo para garantizar una hemodiálisis (HD) eficiente, con unos índices dialíticos (KT) adecuados y conseguir la estabilidad clínica del paciente con ERCT tratado con HD ${ }^{1}$.

Existen dos tipos diferentes de técnicas de canulación de la fístula arteriovenosa nativa (FAVn $)^{2-6}$ :

1. Técnica de la punción escalonada o rotatoria (aguja de punta afilada). Es el método de punción de elección para la mayoría de los pacientes. Las punciones se distribuyen regularmente a lo largo de toda la longitud de la vena arterializada de la FAVn. En cada sesión de HD, se escogen 2 nuevos sitios para la colocación de la aguja permitiendo de este modo la cicatrización de la piel entre las sesiones de HD. Con esta técnica, se produce un aumento moderado del diámetro venoso a lo largo de toda la longitud de la vena arterializada con ausencia o desarrollo mínimo de aneurismas (se evita el debilitamiento progresivo de la pared de la vena secundario al flujo de retorno sanguíneo si incide en el mismo punto). El problema 
principal es que se requiere una vena arterializada que tenga un trayecto suficientemente largo para permitir esta punción rotatoria.

2. Técnica del área o de la punción circunscrita en la misma área (aguja de punta afilada). Los motivos principales para el uso de este método son: longitud limitada de la vena arterializada, ruta difícil para la canulación, miedo de la enfermera de errar en la punción y preferencia del paciente. Esta técnica consiste en la punción repetida en una zona muy circunscrita de la vena arterializada lo que produce la lesión de la pared venosa con la formación de aneurismas. Por tanto, este método debe evitarse siempre que sea posible. Sin embargo, la situación actual en el "mundo real" es decepcionante. Según una encuesta internacional realizada en 171 centros de HD sobre 10.807 canulaciones con dos agujas en pacientes dializados mayormente por FAVn $(91 \%)$, la técnica utilizada con mayor frecuencia (61\%) fue el método del área ${ }^{7}$.

La ecografía Doppler (ED) es una técnica de imagen no invasiva que permite la exploración de la FAVn mediante un transductor lineal emisor y receptor de ultrasonidos aplicado con un ángulo de incidencia $\leq 60^{\circ}$ en los planos longitudinal y transversal de la misma. Desde hace varios años se recomienda la introducción de la ED en todas las Unidades de HD de España ${ }^{8}$. Se han descrito las siguientes prestaciones de la ED?:

1. Método de diagnóstico rápido utilizado in situ en la sala de HD (ecógrafo portátil) ante cualquier disfunción detectada en la FAVn.

2. Método reglado de vigilancia para la evaluación periódica de la FAVn.

3. Información funcional mediante la determinación del flujo (Qa) de la FAVn.

4. Prueba de imagen de elección para confirmar, localizar y cuantificar una estenosis de la FAVn antes de efectuar el tratamiento correctivo.

5. Canulación exitosa de FAVns dificultosas y, por tanto, permite reducir los errores en la punción.

El objetivo del estudio fue identificar a los pacientes portadores de una FAVn que se puncionan mediante la técnica del área, y valorar los hallazgos obtenidos mediante la exploración por ED, para cambiarlos por la técnica escalonada.

\section{Pacientes y método}

El diseño del estudio consiste en un estudio retrospectivo, transversal y observacional, el periodo del estudio fue febrero del 2016.

Las fístulas arteriovenosas nativas (FAVns) analizadas fueron autologas en territorio humeral y radial, con canalización de la vena arterializada mediante bipunción con técnica de área durante 12 sesiones de HD consecutivas, con flujo sanguíneo efectivo (QC) superior a 300 $\mathrm{ml} / \mathrm{min}$ durante 12 sesiones de HD consecutivas como mínimo, y sin evidencia de estenosis.

El ámbito del estudio fue la unidad de Hemodiálisis del área de Nefrología del Hospital de Mollet. El tipo de técnica utilizada fue la HD convencional, a excepción de un paciente que utilizaba la técnica de hemodiafiltración (HDF), del cual solo se usaron las variables que no estaban afectadas por el cambio de técnica.

Todos los enfermos realizaron sesiones de cuatro horas, a excepción de dos que realizaron menos tiempo, de estos también fueron desechadas las variables afectadas por el tiempo.

La variable principal del estudio fue la técnica de punción, el resto de variables fueron del AV ( tipo de AV, distancia entre las agujas, duración del AV y número total de AV), de la vena arterializada del AV ( determinación del flujo de la arteria nutricia mediante ED, profundidad de la vena arterializada determinado por ED y diámetro de la vena arterializada determinado por ED), y por ultimo las variables propias del paciente (edad del paciente, en el momento de su inclusión, género, talla, índice de masa corporal, etiología de la ERCT, tiempo en programa de HD, antecedentes de cardiopatía isquémica, antecedente de artropatía periférica y antecedente de enfermedad cerebrovascular).

El desarrollo del estudio se realizó mediante los siguientes pasos: efectuar la historia clínica y la exploración física del paciente, identificar a los pacientes que cumplen los criterios de inclusión y de exclusión, entregar la hoja de información y obtención del consentimiento informado, exploración de la FAVn mediante ED según la práctica clínica habitual de la Unidad de HD y cumplimentar en una hoja de recogida de datos de todas las variables basales propias del paciente y del AV.

Para el análisis estadístico se utilizó el programa SPSS 13.0 para Windows, donde los valores se calcularon y expresaron como porcentajes, media y desviación estándar. 


\section{Resultados}

Dentro de nuestra población de hemodiálisis tenemos, en el momento que se efectúa el estudio, un total de 63 pacientes prevalentes, 24 de ellos fueron fístulas arteriovenosas protésicas (FAVp) y catéteres, 39 de accesos FAVns y de ellos 9 con punción en área $(14,4 \%)$, el resto en punción escalonada $(85,6 \%)$.

Con una $n=9$ pacientes, sobre las variables basales tenemos: 9 FAVns humerales con técnica de punción en área (6 humerobasilica y 3 humeroperforante), media de edad de 73 , el $55,5 \%$ fueron mujeres, el tiempo medio en HD ( meses) fue 53,6 26,9 , el índice de masa corporal (IMC) fue $24,3 \pm 4,3$, la nefropatía primaria fue ( nefropatía intersticial 33,3\%, amiloidosis $11,1 \%$, no filiada $44,4 \%$ y diabetes mellitus $11,1 \%$ ), con antecedentes cardiopatía isquémica $46,7 \%$, diabetes mellitus $20 \%$, antecedentes de accidente vascular cerebral $6,7 \%$, con al menos una comorbilidad el $77,7 \%$, el número total de AV previos fue $1,8 \pm 1,1$, la duración media de las FAVns actuales ( meses) fue de fue $60,9 \pm 68,4$ y la distancia entre agujas $(\mathrm{cm})$ fue $4,0 \pm 2,1$. (Tabla 1).

Tabla 1.

RESULTADOS $\mathbf{N}=9$

\begin{tabular}{l|c}
\hline Edad & $73 \pm 13,3$ \\
\hline Sexo & $H: 55,5 \% \quad M: 44,5 \%$ \\
\hline Tiempo en HD (meses) & $53,6 \pm 26,9$ \\
\hline Índice de masa corporal & $24,3 \pm 4,3$ \\
\hline Nefropatía primaria & intersticial: $33,3 \%$, amiloidosis: $11,1 \%$, no filiada: $44,4 \%$, diabetes mellitus: $11,1 \%$ \\
\hline Antecedente arteriopatía isquémica & $46,70 \%$ \\
\hline Antecedente arteriopatía periférica & $26,70 \%$ \\
\hline Diabetes & $20 \%$ \\
\hline Antecedente accidente vascular cerebral & $6,70 \%$ \\
\hline FAV/n Radial/Humerral & $(0) 0 \% /(9) 100 \%$ \\
\hline 1 comorbilidad mínimo & $77,70 \%$ \\
\hline Humerocefálica/Humerobasílica/Humeroperforante & $(0) 0 \% /(6) 66,6 \% /(3) 33,3 \%$ \\
\hline Número totales de AV previos & $1,8 \pm 1,1$ \\
\hline Tiempo FAVn acutal (meses) $60,9+68,4$ & $4 \pm 2,1$ \\
\hline Distancia en agujas (cm) & \\
\hline
\end{tabular}

Los resultados de los parámetros ecográficos tenemos una media de diámetro $0,7 \pm 0,3 \mathrm{~cm}$ y $0,5 \pm 0,3 \mathrm{~cm}$ de profundidad. El flujo medio fue de $1309 \pm 966 \mathrm{ml} /$ minuto.

Tabla 2. Parámetros de la exploración ecográfica.

\begin{tabular}{l|c|c|c}
\hline PACIENTES & DIÁMETRO (cm) & PROFUNDIDAD (cm) & FLUJO (ml/min) \\
\hline PACIENTE 1 & $1,41 \pm 0,28$ & $0,36 \pm 0,02$ & 2289 \\
\hline PACIENTE 2 & $0,64 \pm 0,04$ & $0,55 \pm 0,11$ & 605 \\
\hline PACIENTE 3 & $0,70 \pm 0,75$ & $0,59 \pm 0,08$ & 1944 \\
\hline PACIENTE 4 & $0,52 \pm 0,05$ & $0,39 \pm 0,05$ & 480 \\
\hline PACIENTE 5 & $0,37 \pm 0,02$ & $0,58 \pm 0,06$ & 460 \\
\hline PACIENTE 6 & $0,56 \pm 0,56$ & $0,24 \pm 0,07$ & 1.561 \\
\hline PACIENTE 7 & $0,77 \pm 0,04$ & $0,26 \pm 0,05$ & 3100 \\
\hline PACIENTE 8 & $0,77 \pm 0,11$ & $1,07 \pm 0,25$ & 759 \\
\hline PACIENTE 9 & $0,67 \pm 0,02$ & $0,78 \pm 0$ & 519 \\
\hline TOTAL & $0,7 \pm 0,3$ & $0,5 \pm 0,3$ & $1.309,9 \pm 0966$ \\
\hline
\end{tabular}

En siete enfermos fue posible utilizar directamente la técnica escalonadagraciasalaampliación de la zona puncionable de la vena arterializada utilizando la ED. En dos pacientes, fue imprescindible la superficialización previa de la misma. Por tanto, todos los casos de técnica del área pueden ser potencialmente cambiados por la punción escalonada. 


\section{Discusión}

El artículo de Gualy y cols. ${ }^{6}$ nos habla de la importancia del uso adecuado del AV y de su efecto directo sobre la dosis de HD, en la misma línea nos encontramos a Loon ${ }^{3}$ y Dinwiddie y cols. ${ }^{4}$, los cuales explican las complicaciones de los problemas de canulación que sufre el $\mathrm{AV}$, todos estas complicaciones secundarias a su manipulación pueden ser corregidas con el uso del $E D$, esto nos permitiría encontrar nuevas zonas de punción, que fuesen más seguras y con una distancia más adecuada, ello se traduciría en una reducción de la tasa de recirculación y una mejora de la dosis de HD, esto es posible por la interpretación de los datos que nos proporciona el ED sobre: profundidad, diámetro, y dirección del flujo( ecodoppler color).

Por otro lado Roca-Tey R., en su artículo sobre el acceso vascular en el paciente anciano ${ }^{9}$, nos comenta la importancia de la personalización del tratamiento de los pacientes mediante la planificación y monitorización del $A V^{8}$, y en concreto del paciente anciano, de cómo es posible aumentar su esperanza de vida mediante un AV viable y usado adecuadamente, alargando su vida útil y evitando la implantación de catéteres, que por sus complicaciones secundarias, reducen la esperanza de vida.

El uso del ED en el AV del paciente anciano sirve de complemento clave para planificación de la construcción y el uso del AV de forma óptima.

Además la monitorización del AV es básica para la supervivencia del mismo como se afirma en el artículo sobre las mejoras del estado actual de los $\mathrm{AV}^{7}$, en concreto dentro de los conceptos básicos de mejora, cabe recalcar, la introducción del mapa vascular en las consultas de enfermedad renal crónica avanzada (ERCA), y la introducción del ED portátil en la sala de HD, estos conceptos clave se basan en el uso del ED. Nosotros en nuestro artículo además de estar de acuerdo, creemos que utilizar el ED en sala después de la construcción de las FAVns para buscar estratégicamente nuevos puntos de punción, es muy útil para conseguir que las punciones se puedan realizar con la técnica escalonada, en vez de área.

Por último el estudio de Rueda Velasco L. y cols. ${ }^{10}$ se recomienda, sobre la introducción por parte de enfermería del ED en la punción del acceso vascular, la formación en su uso. Las conclusiones de su estudio, nos dicen que el ED es una herramienta sumamente útil para las punciones dificultosas, recanalización de agujas, primeras punciones, localización del bisel de la aguja, todo ello en pro de que enfermería pueda minimizar las complicaciones secundarias a las punciones del AV.

También en el apartado de resultados dice "también es útil esta herramienta para cambiar zonas de punción ", con nuestro estudio hemos querido llegar más lejos, y buscar nuevas zonas potencialmente viables y factibles de punción (puncionables directamente o después de su superficialización pertinente), esto nos ha permitido aprovechar todo un capital venoso arterializado sin uso, y nos ha dado la posibilidad de cambiar potencialmente las zonas de punción, designando nuevos puntos, y por consiguiente poder cambiar la técnica de punción en área, por la técnica de punción escalonada.

Twardowski comenta en un artículo sobre actualización de las técnicas de punción ${ }^{5}$, que las complicaciones que sufre el AV a lo largo de su vida útil, impiden que siempre se utilice la técnica de punción escalonada en los mismos, y pensamos que la ED nos puede permitir llevar a cabo la técnica escalonada en la gran mayoría de estos casos mediante un abordaje del AV utilizando este recurso.

\section{Conclusiones}

1) Se ha registrado una baja incidencia de utilización de la técnica de punción en área en nuestra Unidad de HD.

2) Según los resultados de nuestro estudio, es factible cambiar la técnica de punción en área por escalonada en todos los casos a partir de los parámetros morfológicos y funcionales obtenidos en la ED.

3) La ED portátil utilizada "in situ" en la sala de HD es una herramienta valiosa que permite optimizar la técnica de punción de la FAVn.

\section{Agradecimientos}

Agradecer al Área Nefrológica del Hospital de Mollet, a los pacientes del estudio, por ayudar, participar y contribuir a la realización de este estudio. 
Recibido: 30 agosto 2016

Revisado: 25 septiembre 2016

Modificado: 23 octubre 2016

Aceptado: 30 octubre 2016

\section{Bibliografía}

1. Roca-Tey R. Control de los accesos vasculares. Exploración física e instrumental. En: María Teresa González Álvarez, Román Martínez Cercós: "Manual de accesos vasculares para hemodiálisis". Marge Médica Books, Barcelona, España. Primera edición, 2010, 87-97.

2. Vachharajani TJ. The Role of Cannulation and Fistula Care. Seminars in Dialysis 2015; 28: 24-27.

3. Loon, M. M. (2011). Cannulation practice and complications in hemodialysis vascular access (Doctoral dissertation, Maastricht university).Gallieni $M$, Brenna I, Brunini F, Mezzina N, Pasho S, Fornasieri. Which cannulation technique for which patient. J Vasc Access 2014;15 (Suppl 7): S85-S90.

4. Dinwiddie LC, Ball L, Brouwer D, Doss-McQuitty S, Holland J. What Nephrologists Need to Know about Vascular Access Cannulation. Seminars in Dialysis 2013; 26: 315-322.

5. Twardowski ZJ. Update on cannulation techniques. J Vasc Access 2015; 16 (Suppl 9): S54-S60.

6. Gauly A, Parisotto MT, Skinder A, Schoder V, FurIan A, Schuh E, Marcelli D. Vascular access cannulation in hemodialysis patients - a survey of current practice and its relation to dialysis dose. J Vasc Access. 2011;12:358-64.

7. Roca-Tey R. El acceso vascular para hemodiálisis: la asignatura pendiente. Nefrología 2010;30:280287.

8. Roca-Tey R. Vigilancia y seguimiento del acceso vascular: indicaciones, cuidados y métodos de monitorización. Situación actual del acceso vascular en Cataluña. En: "Avances en diálisis". Collado S, Pascual J (coord.). Barcelona: Publicaciones Permanyer; 2012. p. 49-59.

9. Roca-Tey, R. El acceso vascular del paciente anciano en programa de hemodiálisis. Nefrología (2012). 3(6): 13-20.

10. Rueda Velasco, L., Martín Cano, M., \& Galiano Roa, R. F. (2015). Eco-doppler como herramienta en la punción de fistulas arterio-venosas. Enfermería Nefrológica;18 (3): 110-110. 